\title{
On the Convergence of Logistic Map in NOOR Orbit
}

\author{
Renu Chugh \\ Department of Mathematics \\ M. D. University, Rohtak \\ 124001, INDIA
}

\author{
Mamta Rani \\ Department of MCA \\ Krishna Engg. College \\ Ghaziabad, INDIA
}

\author{
Ashish \\ Department of Mathematics \\ M. D.University, Rohtak \\ 124001, INDIA
}

\begin{abstract}
The logistic map $r x(1-x)$ was given by the Belgian mathematician Pierre Francois Verhulst around 1845 and worked as basic model to study the discrete dynamical system. The behavior of logistic map has been already studied in orbits of one-step, two-step and three-step iterative procedures and it has been established that the logistic map is convergent for larger values of ' $r$ ' for two-step and three-step iteration methods. In this paper, an attempt have been made to study the convergence of logistic map in Noor orbit, which is a four-step iterative procedure.
\end{abstract}

\section{Keywords}

Logistic map, Picard orbit, Noor orbit.

\section{INTRODUCTION}

On November 30, 1844, at the age of 40, when Pierre Francois Verhulst presented his contribution to the "Memoires de L'Academie" of the young Belgian nation, which was published in "tome XVIII" with the title "mathematical investigation of the law of population growth", he did certainly not know that his work would be the starting point for further research in the field of mathematical biology, biometry, and demography. It was in these Memories that

he introduced a growth model for a closed population facing a living environment with limited resources for the subsistence of its members. The purpose was to predict the demographic evolution of the young Belgian society and to answer the question about the maximum population size sustainable with these limited resources. He certainly did not expect that more than one century later, the study of a discrete version of this model would give rise to a new field in science: chaos theory (see [21]).

The logistic equation (some times called the Verhulst Model or logistic growth curve) $\mathrm{x}+\mathrm{rx}(1-\mathrm{x})$ is a model of population growth first published by P.F. Verhulst $(1845,1847)$. The model is continuous in time, but a modification of the continuous equation to a discrete quadratic recurrence equation known as the logistic map. Logistic map is obtained by removing the first term from logistic equation. In its more usual form logistic map is written as $\mathrm{x}=\mathrm{rx}(1-\mathrm{x})$, where $\mathrm{x}$ lies between zero and one and ' $r$ ' is a positive real number. Logistic map plays an important role in the applications of pure and applied mathematics. For a detailed study on logistic map one may refer to Aiden [1], Devaney [3], Holmren [4], M.S.EL Naschie [9-12], Peitgen, Jurgens and Saupe [15] etc. Recently, cryptography has emerged as a major application of logistic map (see for instance [5], [7], [14] and [24]). A comparative study of the logistic map in Picard orbit and that of Norlund orbit is giving by Kumar and Rani [6]. Nowadays the logistic map is studied intensively as an archetypal one dimensional dynamical system.

The one-dimensional logistic map has been extensively studied in the past years. This map describes the typical behavior of many dissipative dynamical systems and has application in Physics, biology, electronics and many other different subjects. Wackerbaur et al [23] detected behavior of logistic map on the basis of different measures of complexity. Salarich and Alasfy [21] utilized the entropy control technique for chaos elimination and presented the logistic map as a case study for the same. Song and Meng [22] proved synchronization in logistic mapping is studied using absolute value feedback control. Molina et al [8] gave embedding dimensions of time series in logistic map and other chaotic maps. Rani and Agarwal [17] generated Julia set by logistic map and studied their distortion under the effect of Noise.

In 2005, Rani and Kumar [20] studied logistic map via new approach. They introduced the superior orbit as an example of two-step iterative procedure and showed that the logistic map is convergent for the large value of ' $r$ ' as compared to Picard orbit. Recently, Rani and Goel studied the convergent behavior [18] and stability [19] of the logistic map via Ishikawa iterates. They found that the map is more stable in Ishikawa orbit than that of Picard orbit.

In this paper, the main tool is Noor iterative procedure. Here, $\mathrm{N}$-orbit stands for the orbit generated by Noor iterative procedure [13]. It is proposed to study the convergent behavior of logistic map in $\mathrm{N}$-orbit. In Section 2 basic definitions and a few parameters that have been taken into account during our study have been described. In Section 3 main result have been presented, followed by concluding remarks in Section 4.

\section{PREMILANIRIES}

Logistic map have been studied using Picard iterative procedure. Following is the definition of Picard orbit:

Let us consider $(X, d)$ be a linear metric space and $T$ is a self mapping from $\mathrm{X}$ into $\mathrm{X}$.

Definition 2.1[16]. Let $\mathrm{X}$ be a non-empty set and $f: \mathrm{X} \rightarrow \mathrm{X}$. For a point $x_{0}$ in $\mathrm{X}$, the Picard orbit (generally called orbit of $f$ ) is the set of all iterates of a point $x_{0}$, i.e., 


$$
O\left(f, x_{0}\right)=\left\{x_{\mathrm{n}}: x_{\mathrm{n}}=f x_{\mathrm{n}-1}, \mathrm{n}=1,2, \ldots\right\} .
$$

where the $O\left(f, x_{0}\right)$ of $f$ at the initial point $x_{0}$ is the sequence $\left\{f^{\mathrm{n}} x_{0}\right\}$.

Definition 2.2. Let us consider a sequence $\left\{x_{n}\right\}$ of iterates for initial point $x_{0} \in \mathrm{X}$ such that,

$N O\left(T, x_{0}, \beta_{\mathrm{n}}, \alpha_{\mathrm{n}}, \gamma_{\mathrm{n}}\right)=\left\{x_{\mathrm{n}+1}: x_{\mathrm{n}+1}=\left(1-\beta_{\mathrm{n}}\right) x_{\mathrm{n}}+\beta_{\mathrm{n}} T y_{\mathrm{n}} ;\right.$

$y_{\mathrm{n}}=\left(1-\alpha_{\mathrm{n}}\right) x_{\mathrm{n}}+\alpha_{\mathrm{n}} T z_{\mathrm{n}}$

$\left.z_{\mathrm{n}}=\left(1-\gamma_{\mathrm{n}}\right) x_{\mathrm{n}}+\gamma_{\mathrm{n}} T x_{\mathrm{n}} ; \mathrm{n}=0,1,2, \ldots\right\}$

where $\alpha_{\mathrm{n}}, \beta_{\mathrm{n}}, \gamma_{\mathrm{n}} \in[0,1]$ and $\left\{\alpha_{\mathrm{n}}\right\},\left\{\beta_{\mathrm{n}}\right\},\left\{\gamma_{\mathrm{n}}\right\}$ are sequences of positive numbers, which is called as $N$-orbit $(N O)$. The above sequence of iterates is called as Noor iterates [15]. Also when $\gamma_{\mathrm{n}}=\beta_{\mathrm{n}}=0$ and $\alpha_{\mathrm{n}}=1$ then it behaves as Picard orbit. In the computations, $\alpha_{\mathrm{n}}=\alpha, \beta_{\mathrm{n}}=\beta, \gamma_{\mathrm{n}}=\gamma, \mathrm{n}=0,1,2, .$. have been taken.

\section{A COMPUTATIONAL APPROACH TO THE STUDY OF LOGISTIC MAP IN $N O$ ( $N$-ORBIT)}

In the computational study, a program have been generated in Mathematica for $\alpha=\beta=\gamma$ chosen between zero and one (i.e. $\alpha=\beta=\gamma \in[0,1]$ ). Let $x_{0}$ be the point in $\quad[0,1]$, we attempt to find the maximum value of ' $r$ ' correctable up to two decimal places, for which the logistic map shows the convergent behavior to a fixed point.

Now, we present some graphical representations of $N O$ using time series representation, which shows the optimum value of ' $r$ ' for the specific choices of $\alpha=\beta=\gamma$.

(i)

Table: 1

For $\alpha=\beta=\gamma=0.9$

\begin{tabular}{|c|c|}
\hline$x_{0}$ & $r$ \\
\hline 0.15 & 3.11 \\
\hline 0.25 & 3.11 \\
\hline 0.35 & 3.11 \\
\hline 0.50 & 3.11 \\
\hline
\end{tabular}

Table 1 shows that the logistic map is convergent at $\alpha$ $=\beta=\gamma=0.9$ for $0<r \leq 3.11$, for all $x \in[0,1]$. See the convergent behavior of map in $N O$ in time series diagram given in Fig. 1. For $r>3.11$ the logistic map shows the nonconvergent behavior in $N O$. Since the non - convergent behavior at $r=3.12$ is negligible (not observable). So, the Fig. 2 shows the non-convergent behavior for $\mathrm{r}=3.70$ at $\quad \alpha=\beta$ $=\gamma=0.9$.

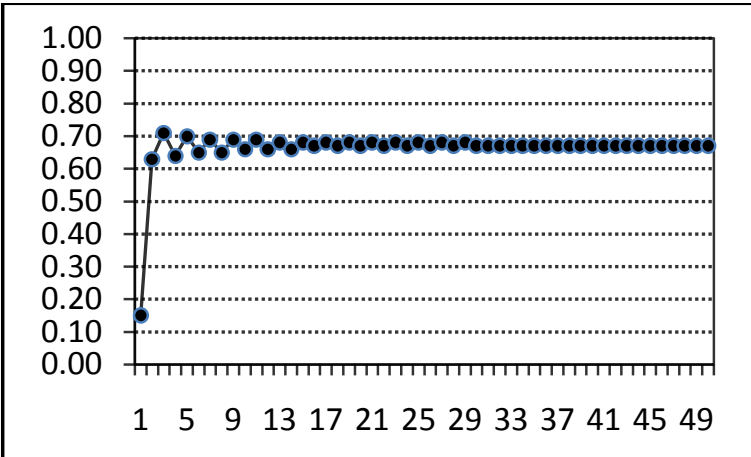

Fig. 1: Convergent behavior of logistic map at $\left(\alpha, \beta, \gamma, r, x_{0}\right)=(0.9,0.9,0.9,3.11,0.15)$

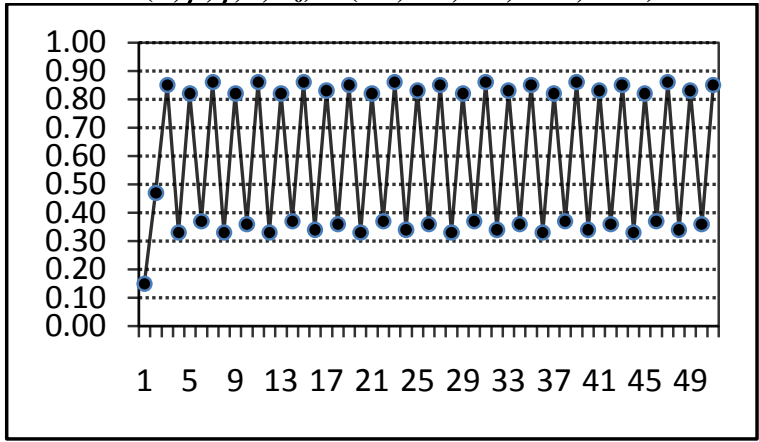

Fig. 2: Non-Convergent behavior of logistic map at (ii) $\left(\alpha, \beta, \gamma, r, x_{0}\right)=(0.9,0.9,0.9,3.70,0.15)$

Table: 2

\begin{tabular}{|c|c|}
\multicolumn{2}{c}{ For $\alpha=\beta=\gamma=0.44$} \\
\hline$x_{0}$ & $r$ \\
\hline 0.15 & 4.67 \\
\hline 0.25 & 4.67 \\
\hline 0.35 & 4.67 \\
\hline 0.50 & 4.68 \\
\hline
\end{tabular}

Table 2 shows that for $\alpha=\beta=\gamma=0.44$ and $0<r \leq 4.67$, for all $x_{0} \in[0,1]$ the logistic map is convergent to a fixed point. So, the Fig. 3 shows the convergence of the logistic map in $N O$ for $r=3.00$ at $x_{0}=0.35$

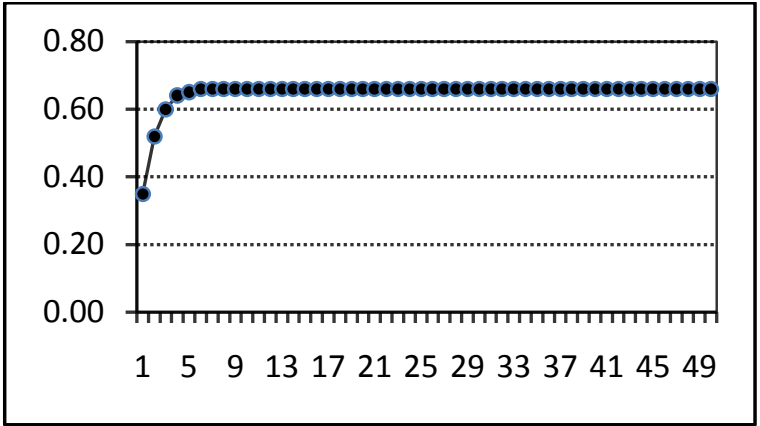

Fig. 3: Convergent behavior of logistic map at $\left(\alpha, \beta, \gamma, \boldsymbol{r}, \boldsymbol{x}_{\mathbf{0}}\right)=(\mathbf{0 . 4 4 , 0 . 4 4 , 0 . 4 4 , 3 . 0 0 , 0 . 3 5 )}$ 
(iii)

Table: 3

\begin{tabular}{|c|c|}
\multicolumn{2}{c}{ For $\alpha=\beta=\gamma=0.1$} \\
\hline$x_{0}$ & $r$ \\
\hline 0.15 & 15.00 \\
\hline 0.25 & 15.01 \\
\hline 0.35 & 15.05 \\
\hline 0.50 & 15.08 \\
\hline
\end{tabular}

In Table: 3 the logistic map shows the convergent behavior for $0<r \leq 15.00$ at $\alpha=\beta=\gamma=0.1$. For $r>15.00$ at $\quad x_{0}=0.15$, logistic map either shows the cyclic behavior or undefined behavior.

(iv)

Table: 4

For $\alpha=\beta=\gamma=0.05$

\begin{tabular}{|c|c|} 
For $\alpha=\beta=\gamma=0.05$ \\
\hline$x_{0}$ & $r$ \\
\hline 0.15 & 28.44 \\
\hline 0.25 & 27.52 \\
\hline 0.35 & 28.86 \\
\hline 0.50 & 28.37 \\
\hline
\end{tabular}

\begin{tabular}{|l|l|}
\hline Convergence in $\boldsymbol{P O}$ & Convergence in $\boldsymbol{N O}$ \\
\hline$\beta=1, \alpha=\gamma=0,0 \leq r \leq 2.75$ & $\alpha=\beta=\gamma=0.9,0 \leq r \leq 3.11$ \\
\hline & $\alpha=\beta=\gamma=0.44,0 \leq r \leq 4.67$ \\
\hline & $\alpha=\beta=\gamma=0.1,0 \leq r \leq 15.00$ \\
\hline & $\alpha=\beta=\gamma=0.05,0 \leq r \leq 27.52$ \\
\hline
\end{tabular}

In Table 4 , for $\alpha=\beta=\gamma=0.05$ and $0<r \leq 27.52$, for all $x_{0} \in[0$, 1] the logistic map is convergent to a fixed point. Fig. 4 shows the convergent behavior to a fixed point when the given map is iterated in $N O$ at $r=27.52$ and $\alpha=\beta=\gamma=0.05$. For $r>27.52$ it shows the undefined behavior of the iterated function in $N O$.

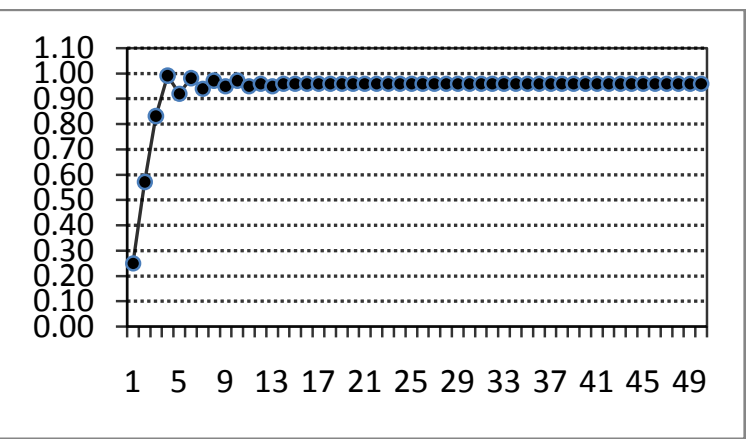

Fig. 4: Convergent behavior of logistic map at $\left(\alpha, \beta, \gamma, \boldsymbol{r}, \boldsymbol{x}_{\mathbf{0}}\right)=(\mathbf{0 . 0 5}, \mathbf{0 . 0 5}, \mathbf{0 . 0 5}, \mathbf{2 7 . 5 2}, \mathbf{0 . 1 5})$

The above computations shows that when the value of $\alpha=\beta=$ $\gamma$ decreases then the value of ' $r$ ' increases to a specific range upto the convergence of logistic map to a fixed point. Further, we studied the relationship between the parameters $\alpha$ $=\beta=\gamma$ and ' $r$ ' using the graphical representation (see Fig. 4) and introduced the cubic poynomials $\mathrm{y}=0.967 \mathrm{x}^{3}-5.16 \mathrm{x}^{2}+$
$9.477 \mathrm{x}-2.155$ and $\mathrm{y}=0.016 \mathrm{x}^{3}-0.1 \mathrm{x}^{2}-0.116 \mathrm{x}+1.1$ which shows the increment behavior of Series1 and the corresponding decrement behavior in the Series respectively.

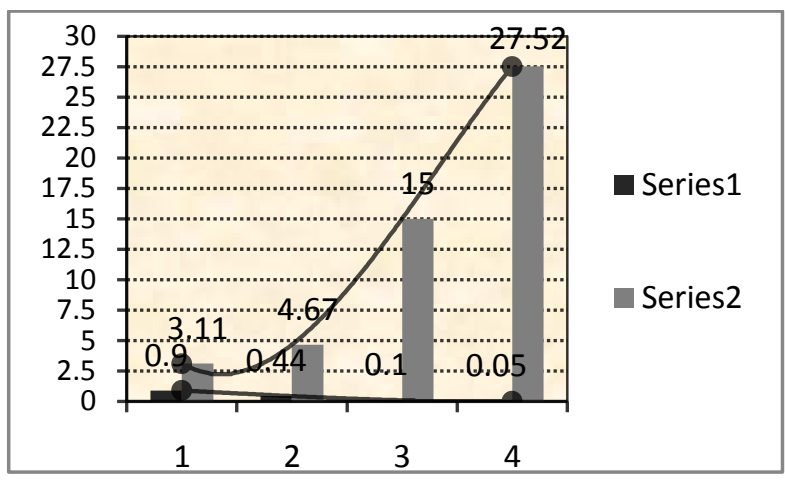

Fig. 5 Showing the relation between $\alpha=\beta=\gamma$ and ' $r$ '

\section{CONCLUSION}

In this paper using computational study, it is concluded that the logistic map is convergent in $N O$ for larger values of ' $r$ ' for all $x \in[0,1]$ than $P O$. Following table gives the comparison of convergence of logistic map in $P O$ and $N O$.

\section{ACKNOWLEDGMENTS}

This research is supported by the University Grant Commission of India (Grant No. 39-29/2010(SR))

\section{REFERENCES}

[1] M. Aidan, J. G. Keating and D. M. Heffernan, A detailed study of the generation of optically detectable watermarks using the logistic map, Chaos Solitons Fractals (2006)(30)(5), pp.1088-1097.

[2] M. Ausloos and M. Dirickx, The Logistic Map and the Routeto Chaos: From the beginnings to Modern Applications, Springer Verlag, New York, 2005. Zbl 1085.37001

[3] R. L. Devaney, A First Course In Chaotic Dynamical Systems: Theory And Experiment, Addison-Wesley, MA, 1992. MR1202237

[4] R. A. Holmgren, A First Course in Discrete Dynamical Systems, Springer-Verlag, New York, 1994. MR1269109

[5] A. Kanso and N. Smaoui, Logistics chaotic maps for binary numbers generations, Chaos Solitons Fractals (2007), doi:10.1016/j.chaos.2007.10.049.

[6] M. Kumar and M. Rani, An experiment with summability methods in the dynamics of the logistic model, Indian J. Math (2005)(47)(1), pp. 77-89. MR2155130

[7] B. Mi, X. Liao and Y. Chen, A novel chaotic encryption scheme based on arithmetic coding, Chaos Solitons Fractals (2008)(38)(5), pp. 1523-1531.

[8] C. Molina, N. Sampson, W. J. Fitzgerald and M. Niranjan, Geometrical techniques for finding the embedding dimension of time series, Proc. IEEE Signal Processing Society Workshop (1996), p.p.161-169. 
[9] M. S. EL Naschie, Nuclear Spacetime Theories, Superstrings, Monster Group and Applications, Chaos Solitons Fractals(1999)(10)(2-3), pp. 567-580.

[10] M. S. EL Naschie, On the universality class of all universality classes and E-infinity space time physics, Chaos Solitons Fractals(2007)(32)(3), pp. 927-936.

[11] M. S. EL Naschie, Super symmetry, transfinite neural networks, hyperbolic manifolds, quantum gravity and the Higgs, Chaos Solitons Fractals (2004)(22)(5), pp. 9991006.

[12] M. S. EL Naschie, Topics in the mathematical physics of E-infinity theory, Chaos Solitons Fractals (2006)(30)(3), pp. 656-663.

[13] M. A Noor, New approximation schemes for general variational inequalities, J. Math. Anal. Appl., 251(2000), 217-229.

[14] L. P. L. de Oliveira and M. Sobottka, Cryptography with chaotic mixing, Chaos Solitons Fractals (2008)(35)(3), pp. 466-471.Zbl 1139.94005 MR2359835

[15] H. O. Peitgen, H. Jurgens and D. Saupe, Chaos And Fractals: New Frontiers of Science, Springer-Verlag, New York, 2004. MR2031217

[16] M. Rani, Ph.D, Thesis, Iterative procedures in fractal and chaos, Gurukala Kangri Vishwavidyala, Hardwar, 2006.
[17] M. Rani and R. Agarwal, Effect of Noise on Julia sets generated by Logistic map, 978-1-4244-5586-7/10, 2010 IEEE, Vol 2.

[18] M. Rani and S. Goel, An experimental approach to study the logistic map in I-superior orbit, Chaos and Complexity Letters, Vol. 5, Iss. 2 (2011), pp. 1-7.

[19] M. Rani and S. Goel, I-Superior approach to study the stability of logistic map, International conf. on Mec. And Elec. Tech. (ICMT 2010), IEEE.

[20] M. Rani and V. Kumar, A new experimental approach study the stability of logistic maps, J. Indian Acad. Math. (2005)(27)(1), 143-156.

[21] H. Salarieh and A. Alasty, Stabilizing unstable fixed points of chaotic maps via minimum entropy control, Chaos Solitons Fractals(2008)(37)(3), pp. 763-769.

[22] W. Song and J. Meng, Research on logistic mapping and synchronization, Proc. IEEE Intelligent Control and Automation,(1996)(1), p.p.987-991.

[23] R. Wackerbauer, A. Witt, H. Atmanspacher, J. Kurths and $\mathrm{H}$. Scheingraber, A comparative classification of complexity measures, Chaos Solitons Fractals (1994)(4)(1), pp. 133-173

[24] H. Yang, X. Liao, K. Wong, W. Zhang and P. Wei, A new cryptosystem based on chaotic map and operations algebric, Chaos Solitons Fractals (2008), doi:10.1016/j. chaos.2007.10.046. 